\title{
Sex-specific mitonuclear epistasis and the evolution of mitochondrial bioenergetics, ageing, and life history in seed beetles
}

Mirko Đorđević, ${ }^{1}$ Biljana Stojković, ${ }^{1,2}$ Uroš Savković, ${ }^{1}$ Elina Immonen, ${ }^{3}$ Nikola Tucić, ${ }^{1, \dagger}$ Jelica Lazarević, ${ }^{4, *}$ and Göran Arnqvist ${ }^{3,5, *}$

${ }^{1}$ Department of Evolutionary Biology, Institute for Biological Research, University of Belgrade, Despota Stefana Boulevard 142, Belgrade 11060, Serbia

${ }^{2}$ Institute of Zoology, Faculty of Biology, University of Belgrade, Studentski trg 16, Belgrade 11000, Serbia

${ }^{3}$ Animal Ecology, Department of Ecology and Genetics, Evolutionary Biology Centre, Uppsala University, Norbyvägen 18D SE-752 36, Uppsala, Sweden

${ }^{4}$ Department of Insect Physiology and Biochemistry, Institute for Biological Research, University of Belgrade, Despota Stefana Boulevard 142, Belgrade 11060, Serbia

${ }^{5}$ E-mail: goran.arnqvist@ebc.uu.se

Received May 25, 2016

Accepted October 24, 2016

The role of mitochondrial DNA for the evolution of life-history traits remains debated. We examined mitonuclear effects on the activity of the multisubunit complex of the electron transport chain (ETC) involved in oxidative phosphorylation (OXPHOS) across lines of the seed beetle Acanthoscelides obtectus selected for a short (E) or a long (L) life for more than $>160$ generations. We constructed and phenotyped mitonuclear introgression lines, which allowed us to assess the independent effects of the evolutionary history of the nuclear and the mitochondrial genome. The nuclear genome was responsible for the largest share of divergence seen in ageing. However, the mitochondrial genome also had sizeable effects, which were sex-specific and expressed primarily as epistatic interactions with the nuclear genome. The effects of mitonuclear disruption were largely consistent with mitonuclear coadaptation. Variation in ETC activity explained a large proportion of variance in ageing and life-history traits and this multivariate relationship differed somewhat between the sexes. In conclusion, mitonuclear epistasis has played an important role in the laboratory evolution of ETC complex activity, ageing, and life histories and these are closely associated. The mitonuclear architecture of evolved differences in life-history traits and mitochondrial bioenergetics was sex-specific.

KEY WORDS: Bruchinae, coadaptation, epistasis, evolution of ageing, mitochondria, mtDNA, OXPHOS, senescence, sexual dimorphism.

As the main site of energy production in the eukaryotic cell, and thus a source of damaging oxygen radicals, mitochondria are clearly a nexus for ageing (Harman 1956, 1972; Das 2006; Guarente 2008; Houtkooper et al. 2013). Yet, mitochondrial genetic variation within species has by tradition been considered neutral and nonfunctional. This view contrasts with an increasing number of recent studies that have shown that naturally occurring

*Both the authors shared senior authorship. mitochondrial DNA (mtDNA) haplotypes affect ageing and lifehistory phenotypes (e.g., James and Ballard 2003; Ellison and Burton 2006; Maklakov et al. 2006; Rand et al. 2006; Ballard et al. 2007a; Clancy, 2008; Dowling et al. 2009; Arnqvist et al. 2010; Immonen et al. 2016).

A number of experimental studies have shown that mitochondrial genetic effects are to a large extent expressed through epistatic interactions with the nuclear genome (Dobler et al. 2014; Levin et al. 2014; Wolff et al. 2014). This is perhaps unsurprising, 
given that mtDNA products integrate with nuclear DNA (nDNA) products to jointly form the multisubunit electron transport chain (ETC) in the inner mitochondrial membrane, which uses oxidative phosphorylation (OXPHOS) to feed the reformation of ATP by ATP synthase. Here, the enzyme complexes I, III, IV, and $\mathrm{V}$ of the OXPHOS pathway are composed of both mtDNAand nDNA-encoded proteins, whereas complex II is built by nDNA-encoded proteins alone. In addition, the mitochondrial specific protein translational machinery comprised both mtDNA and nDNA encoded genes (Levin et al. 2014). Because the OXPHOS pathway is the main energy-producing metabolic pathway of the cell and a major source of reactive oxygen species (ROS), any phenotypic effects of mtDNA on ageing must in some way be rooted in the bioenergetic properties of the OXPHOS pathway. Assays of the energy-producing reactions of the ETC can therefore be used to provide mechanistic and biochemical links between genotypes and life-history phenotypes (Das 2006; Ballard and Melvin 2010; Ballard and Pichaud 2014; Wolff et al. 2016).

Evolution of mitochondrial function may derive from selection acting on (1) endocrine signaling pathways that regulate expression of mitochondrial proteins and affect phospholipid fatty acid composition of mitochondrial membrane or (2) genes encoding OXPHOS proteins and other molecules involved in their transcription and translation (Das 2006). Optimal respiratory activity is regulated by both anterograde (nucleus to mitochondria) and retrograde (mitochondria to nucleus) signaling pathways and depends on proper assembly of mtDNA- and nDNA-encoded subunits of ETC complexes. Therefore, mitonuclear coevolution is a predicted result of selection on mitochondrial function (Rand et al. 2004; Ballard and Rand 2005).

In terms of the evolution of mtDNA, one interesting complication derives from the fact that mtDNA are maternally inherited: the efficacy of selection on mtDNA-related OXPHOS function is therefore likely different in males and females (Frank and Hurst 1996; Gemmell et al. 2004). This predicts the evolution of partially sex-specific regulation of nuclear OXPHOS genes (Meiklejohn et al. 2007; Gallach and Betran 2011). Studies of Drosophila provide support for this, showing Y-linked regulation of sex-biased expression of OXPHOS genes (Lemos et al. 2008), that mitonuclear interactions involving sex chromosomes are different from those involving autosomes (Rand et al. 2001), the evolution of sex-specific expression of nuclear OXPHOS gene duplicates (Gallach et al. 2010), and sex-specific transcriptional interactions between mtDNA and nuclear metabolic genes (Innocenti et al. 2011). If the optimal pattern of ageing and life history is sex-specific (Maklakov and Lummaa 2013), we therefore expect that sex-specific mitonuclear epistasis and coadaptation may proceed along partly distinct trajectories in males and females.
Whether mtDNA variation plays any role in the evolution of ageing is, however, still somewhat contentious (Levin and Mishmar 2015). Much of the relevant empirical foundation is built by comparisons between populations, or even species, where a major limitation is that a lack of detailed information of past selection weakens inferences about responses to selection (Arnqvist et al. 2010). Here, phenotypic and genetic dissection of artificial selection or experimental evolution lines provides a potential remedy. Such efforts yield more definite insights into the genetics of phenotypic responses to selection, simply because divergent selection is experimentally imposed and controlled (Schlötterer et al. 2015). In the present study, we analyzed a set of replicated laboratory populations of the seed beetle (A. obtectus) that had been selected for reproduction early $(E)$ or late $(L)$ in life for $>160$ generations (Tucić et al. 1996, 1997). Previous studies of these unique lines have shown that the E/L selection regime has led to marked divergence in ageing, life span, reproductive schedule, resistance to oxidative stress, sensitivity to inhibition of ETC complex I, metabolite composition, body size, growth rate, and several other life-history traits (e.g., Stojković and Savković 2011; Stojković et al. 2011; Lazarević et al. 2012, 2013; Šešlija Jovanović et al. 2014). For example, beetles from L lines live more than twice as long and senesce half as rapidly as do those from E lines (Đorđević et al. 2015). Importantly, we show in an accompanying study that the $\mathrm{L}$ selection lines are now largely fixed for a particular mtDNA haplotype, whereas the $\mathrm{E}$ lines are all polymorphic for two other mtDNA haplotypes, by and of itself suggesting a role for mtDNA in the divergent evolution observed (Stojković et al. 2016). Here, our overall aims are to use mitochondrial bioenergetic assays of mitonuclear introgression lines to test the predictions (1) that mitochondrial function has diverged as a result of divergent selection on life span, (2) that disruption of potentially coadapted mitonuclear combinations impairs mitochondrial function, and (3) that such mitonuclear genetic effects should be sex-specific. We assayed the activity of ETC complex I-IV and used a mitonuclear introgressive crossing scheme to disentangle nuclear, mitochondrial, and mitonuclear genetic effects on divergence in ETC activity and ageing.

\section{Materials and Methods LIFE HISTORY OF THE SEED BEETLE AND GENERAL EXPERIMENTAL CONDITIONS}

Acanthoscelides obtectus is a cosmopolitan pest of stored legumes. The primary host of these seed beetles is the common bean, Phaseolus vulgaris. Females deposit their eggs on bean seeds. Larvae hatch a few days later and burrow directly into the bean. Larval development and pupation are completed entirely within a single seed. Adult beetles emerge from the seeds and are ready to mate after a few hours. Adults are facultative aphagous, 
and obtain all resources required for survival and reproduction during the larval stage. We cultured A. obtectus on common bean seeds ( $P$. vulgaris) in dark incubators at $30^{\circ} \mathrm{C}$. All experiments reported upon here were done under these conditions and no food or water was offered to adults. All seeds used were from one source and were frozen before being used in the experiments.

\section{AGE-SPECIFIC SELECTION LINES}

A base population (B) of $A$. obtectus, from which all selection lines were originally derived, was established in 1983 by mass mating equal numbers of adults from three local subpopulations of A. obtectus collected near Belgrade (Tucić et al. 1996). The base population was maintained at a large size $(N \approx 5000$ individuals) on Phaseolus vulgaris seeds on a 40-day schedule. The base population was thus maintained in the laboratory for three years (27 generations), prior to founding the selection line populations. Experimental populations were derived simultaneously from the base population during 1986 by splitting the beetles into two contrasting selection regimes, one in which phenotypic selection for long life was removed and one in which we selected for long life. Throughout the course of our long-term selection experiments, reproduction was permitted either in only very young beetles ( $\mathrm{E}$ regime) or in individuals at age 10 days and older ( $L$ regime). In the $E$ regime, where fitness was accrued during days 1-2 of adult life, life span beyond two days thus had no effect on fitness. In the $L$ regime, where fitness was accrued after day 10 of adult life, individuals with a life span shorter than 10 days had zero fitness.

Briefly, each E line was started with approximately 400 newly emerged adults (sex ratio approximately 1:1) that were kept together in a rearing jar with an ample supply of seeds for $48 \mathrm{~h}$ to mate and lay eggs at will, after which all adults were removed. Seeds were then incubated until adult emergence, when the next generation was initiated. To establish each generation in the $\mathrm{L}$ selection regime, approximately 1000 females and males were kept together in 10 separate vials (i.e., about 100 individuals per vial) without seeds to depress oviposition. Beetles surviving to day 10 were then introduced into a rearing jar provided with approximately 100 seeds, and any eggs laid prior to day 10 did thus not contribute to the next generation. We founded four replicate lines of each selection regime. The selection lines were maintained on Phaseolus vulgaris seeds in a dark incubator under aphagous conditions at $30^{\circ} \mathrm{C}$ (see Tucić et al. 1996, 1997; Lazarević et al. 2013).

Although the $\mathrm{E}$ and $\mathrm{L}$ lines were derived simultaneously from the base population, the evolution of differences in preadult development time has led to the two types of lines being asynchronous. It has earlier been shown that L lines have reduced early fecundity, elevated late life fecundity, and prolonged life span in comparison to E lines (Tucić et al. 1996). Here, we used beetles from generation 225 of the $\mathrm{E}$ and generation 160 of the
$\mathrm{L}$ lines. To reduce idiosyncratic line-specific variation and the potential impact of any Wahlund effects present among lines, we first amalgamated the four replicate populations within each selection treatment. This was achieved by mixing approximately 300 beetles from each of the four replicate selection lines and propagating the amalgamated four-way cross lines for two generations to reduce any mitonuclear linkage disequilibrium. These four-way crosses (one $\mathrm{E}$ and one $\mathrm{L}$ ) were then used to construct our mitonuclear introgression (MN) lines. Although this design does not allow us to probe variation between replicate lines within selection regimes, it allows a more focused analysis of the effects shared between lines within selection regimes. Our inferences, thus, rely on the fact that all $\mathrm{E}$ beetles share a common and experimentally imposed selection regime which is distinct from that shared by all L beetles, and we interpret consistent differences between $\mathrm{E}$ and $\mathrm{L}$ beetles as reflecting past selection. We note that evolutionary divergence between our $\mathrm{E}$ and $\mathrm{L}$ lines is marked and very well documented (see references above).

\section{CONSTRUCTION OF MITONUCLEAR INTROGRESSION LINES AND SURVIVAL ASSAY}

We collected 50 virgin one-day-old females from each of the two amalgamated lines to conduct the initial backcrossing. These females represented the founders of our MN lines, and each female was mated to five randomly chosen one-day-old males from the opposite selection regime $(1 q \mathrm{E} \times 5 \hat{\jmath} \mathrm{L}$ and $1 q \mathrm{~L} \times 5 \hat{\jmath}$ $E)$. In the $F_{1}$ generation, offspring thus inherited their mtDNA from mothers (maternal selection regime), while nuclear genes were inherited both from mothers $(50 \%)$ and fathers $(50 \%)$ and $\mathrm{F}_{1}$ females with this degree of disassociation were used as a standard base for future introgressions. Virgin $F_{1}$ females of each type were divided into two groups for the $\mathrm{F}_{2}$ crosses. In the first group, five randomly selected females were each backcrossed to a male from the paternal selection regime, to further disassociate mtDNA from the native nuclear genetic background (disrupted crosses). In the second group, five randomly selected females were each backcrossed to a male from the maternal selection regime, aiming to reconstruct the original mitonuclear combination (reconstituted crosses). This backcrossing scheme was repeated for 14 generations, leading to a predicted replacement of more than $99.99 \%$ of the original nuclear genome of each line. Thus, after 14 generations of backcrossing, the following mitonuclear combinations were obtained: EE (mitochondria representative of the E selection regime and reconstituted with an E nuclear complement), LE (L mitochondria with E nuclear), LL (L mitochondria with L nuclear), and EL (E mitochondria with L nuclear), each replicated five times. All experimental assays were done using these five $\mathrm{MN}$ lines within each mitonuclear combination ( $N=20 \mathrm{MN}$ lines).

To enable a formal survival analysis, we set up a mortality assay involving 150 newly emerged virgin beetles, weighed to the 
nearest $0.1 \mathrm{mg}$, per MN line and sex $(N=6000)$. These beetles were kept isolated individually in separate vials without seeds. Mortality was monitored daily.

Some insects are infected by maternally inherited, cytoplasmic $\alpha$-proteobacteria (i.e., Wolbachia) that can confound the results of experiments that aim to investigate mtDNA effects. Wolbachia infections have previously been screened for in many A. obtectus populations but have never been detected (e.g., Kageyama et al 2010; Li et al. 2015). To preclude the possibility that cytoplasmic bacteria were nevertheless present in our selection lines, we screened for the presence of Wolbachia in all lines by a polymerase chain reaction (PCR) assay. We used the Wolbachia-specific primers and protocols developed by O'Neill et al. (1992) to determine the infection status of samples of beetles from all selection lines. We performed PCR using two pairs of primers simultaneously ("76-99 forward" and "1012-994 reverse"), which amplifies a fragment of $\approx 0.9 \mathrm{~kb}$ of rDNA from Wolbachia. As a positive control for the DNA extraction and PCR reaction, we used a Drosophila melanogaster strain known to be infected with Wolbachia. All screened beetles were PCR-negative for Wolbachia, but the positive control was not. We therefore interpret any cytoplasmic effects seen here as mitochondrial in origin, also supported by the fact that the selection regimes are known to harbor distinct mtDNA haplotypes (Stojković et al. 2016).

\section{ACTIVITY OF ETC COMPLEXES}

The activity of ETC complexes was determined in one-day-old virgin adult individuals. All procedures for isolation of mitochondria were carried out at $4^{\circ} \mathrm{C}$. For each $\mathrm{MN}$ line, five samples were prepared per sex by homogenization of five female or male beetles in STE buffer (0.25 M sucrose, 5 mM Tris/HCl pH 7.4, 2 mM EGTA). Homogenization was performed manually in Eppendorf tubes. After low-speed centrifugation at $700 \times g$ for $15 \mathrm{~min}$, the supernatant was centrifuged at $16,000 \times g$ for $20 \mathrm{~min}$. The resulting pellet was resuspended in $200 \mathrm{~mL}$ of STE buffer and stored at $-80^{\circ} \mathrm{C}$.

The activity of complex I was measured as the decrease in absorbance at $600 \mathrm{~nm}$ due to the reduction of DCIP $(2,6-$ dichloroindophenol) at $30^{\circ} \mathrm{C}\left(\varepsilon=19.1 \mathrm{mM}^{-1} \mathrm{~cm}^{-1}\right)$. After oxidation of NADH by complex I, electrons are transferred to the artificial substrate decylubiquinone and then to DCIP as the terminal electron acceptor. The reaction started by adding NADH $(0.2 \mathrm{mM})$ to a reaction mixture containing mitochondrial proteins, $25 \mathrm{mM}$ potassium phosphate buffer $\mathrm{pH}$ 7.6, BSA (3.5 g/L), DCIP $(60 \mu \mathrm{M})$, and decylubiquinone $(70 \mu \mathrm{M})$ and antimycin $\mathrm{A}(1 \mu \mathrm{M})$ dissolved in dimethyl sulfoxide. This procedure results in high rotenone-sensitive activity because decylubiquinone does not accept electrons from other NADH oxidoreductases (Janssen et al. 2007).
The activity of complex II was also measured as the decrease in absorbance at $600 \mathrm{~nm}$ due to the reduction of DCIP at $30^{\circ} \mathrm{C}$. The reaction mixture here contained mitochondrial proteins, $25 \mathrm{mM}$ potassium phosphate buffer $\mathrm{pH} 7.6$, BSA $(1 \mathrm{mg} / \mathrm{mL})$, DCIP $(75 \mu \mathrm{M})$, EDTA $(2 \mathrm{mM})$, succinate $(20 \mathrm{mM})$, ATP $(0.2$ $\mathrm{mM})$, rotenone $(3 \mathrm{mM})$, antimycin $\mathrm{A}(1 \mu \mathrm{M})$, and $\mathrm{KCN}(1 \mathrm{mM})$. Prior to reaction, complex II was activated by $10 \mathrm{~min}$ incubation with succinate (Barrinetos 2002). The reaction started by adding decylubiquinone $(65 \mu \mathrm{M})$. Blanks were measured in the presence of $10 \mathrm{mM}$ malonate.

The activity of complex III was measured as the increase in absorbance at $550 \mathrm{~nm}$ due to reduction of oxidized cytochrome c at $30^{\circ} \mathrm{C}\left(\varepsilon=21.84 \mathrm{mM}^{-1} \mathrm{~cm}^{-1}\right)$. Electron donor was decylubiquinol prepared from decylubiquinone according to the procedure of Luo et al. (2008). The reaction was initiated by addition of decylubiquinol $(50 \mu \mathrm{M})$ to a reaction mixture containing mitochondrial proteins, $25 \mathrm{mM}$ potassium phosphate buffer $\mathrm{pH}$ 7.6, BSA $(0.1 \mathrm{~g} / \mathrm{L})$, Tween $20(0.05 \%)$, sodium azide $(4 \mathrm{mM})$, rotenone (4 $\mu \mathrm{M})$, ATP (0.2 mM), and oxidized cytochrome c (horse heart; $50 \mu \mathrm{M})$. Residual activity after the addition of antimycin A $(1 \mu \mathrm{M})$ was determined simultaneously.

The activity of complex IV was measured by monitoring the decrease in absorbance at $550 \mathrm{~nm}$ due to oxidation of reduced cytochrome $\mathrm{c}$ at room temperature $\left(\varepsilon=21.84 \mathrm{mM}^{-1} \mathrm{~cm}^{-1}\right)$. The reaction started after addition of reduced cytochrome $\mathrm{c}(10 \mu \mathrm{M})$ to a reaction mixture containing assay buffer $(10 \mathrm{mM}$ Tris/ $\mathrm{HCl}$ $\mathrm{pH} 7,120 \mathrm{mM} \mathrm{KCl}$ ) and mitochondrial proteins dissolved in dilution buffer (10 mM Tris/ $\mathrm{HCl} \mathrm{pH}$ 7, $250 \mathrm{mM}$ sucrose, $0.1 \mathrm{mM}$ $n$-dodecyl- $\beta$-D-maltoside). Blanks contained all components except mitochondrial proteins.

Protein content was estimated according to Bradford (1976), using bovine serum albumin as a standard. Specific activities were expressed as enzyme units (U) per mg of proteins. For complexes I, II, and III, one enzyme unit corresponded to the reduction of $1 \mu \mathrm{M}$ of terminal electron acceptors per minute (DCIP for complexes I and II, and oxidized cytochrome $\mathrm{c}$ for complex III). For complex IV one enzyme unit oxidized $1 \mu \mathrm{mol}$ of cytochrome $\mathrm{c}$ per minute. The repeatability of the MN line specific ETC activity measures, estimated as the ratio of among-MN-line variance to total variance, was $0.60,0.74,0.89$, and 0.78 for ETC complex I-IV, respectively.

\section{STATISTICAL ANALYSES}

In all statistical models, the terms "nuclear" (nDNA) and "mitochondrial" (mtDNA) refer to genetic effects that are due to the respective genome having had a history of being selected for early or late life reproduction (see above). We first described sexspecific mortality within MN lines by fitting the three-parameter logistic model $u(x)=a \mathrm{e}^{b x} /\left(1+s^{2} L[x]\right)$, where $u(x)$ is age-specific mortality rate, $x$ is age, $a$ simply is baseline mortality rate, $b$ is 
the exponential increase in mortality with age (i.e., senescence), $s$ is the degree of old-age mortality deceleration, and $L(x)=$ $(a / b)\left(\mathrm{e}^{b x}-1\right)$, using WinModest (Pletcher 1999). We then tested for effects of the evolutionary history of the mitochondria and the nuclear genome, as well as those of sex and body weight (zero centered), on survival in a mixed effects Cox proportional hazards model using the coxme version 2.2-5 package in $\mathrm{R}$ (Therneau 2015). Here, MN replicate line was treated as a random effects factor and other classifiers as fixed effects variables. Variation in life span across individuals was analyzed in a generalized linear mixed effects model (using a Poisson error distribution, an identity link function, and an empirical dispersion parameter) in which mtDNA, nDNA, sex, and their interactions were fixed effects factors and $\mathrm{MN}$ line, nested within mtDNA $\times$ nDNA combination, was treated as a random effects factor.

The effects of sex and the evolutionary history of mitochondrial and nDNA on mitochondrial ETC enzyme activities were analyzed in a series of mixed effects linear models, in which mtDNA, nDNA, sex, and their interactions were fixed effects factors and $\mathrm{MN}$ line, nested within mtDNA $\times$ nDNA combination, was treated as a random effects factor. Models were fitted using REML estimation with type III sums of squares. We tested whether the data fulfilled the assumption made by these inferential models, in terms of normality of errors and homoscedasticity, and found that it did $(P>0.05$ in all cases). We therefore did not apply any transformation of the ETC activity data. The hypothesis of coadaptation between the nuclear and mitochondrial genomes was tested by sex-specific focused $t$ tests, assessing whether ETC activity was affected when a nuclear genome was expressed with a mitochondrial genome with which it shared, or did not share, past selection regime.

The MN lines studied here were also, in an accompanying study (Đorđević et al. 2015), characterized with respect to major life-history traits (i.e., sex-specific development time, body mass, and longevity). Here, we used this opportunity to ask whether, across MN lines, sex-specific mitochondrial ETC activity predicted major life-history traits (data in Tables S1 and S2). This was tested both in a series of general linear models of each of the three life-history traits as well as senescence and old-age mortality, using the four ETC activities, sex and their interactions as predictors, and in a set of multivariate canonical correlation analyses.

\section{Results}

\section{SURVIVAL IN MITONUCLEAR LINES}

Survival and mortality curves are presented for MN line-pooled data in Figure 1 (see also Fig. S1 and Table S2), illustrating strong, and primarily nuclear, genetic effects on age-specific survival rates. Mixed effects Cox proportional hazards mod- eling revealed effects of the evolutionary history of both the nuclear and the mitochondrial genome on survival (Table 1). The addition of body weight and its interactions with the factorial variables to a model including only the factorial variables significantly improved model fit to data $\left(\chi^{2}=712.57\right.$, df $=8, P<0.001)$. Following model simplification by removal of nonsignificant interactions, the model of size-specific survival validated the initial model (Table 1). In short, the effects seen were to a large extent sex-specific, as revealed by significant interactions between sex $\times$ mtDNA and sex $\times$ nDNA on survival. Our analysis of variation in life span corroborated this interpretation. Here, nuclear genetic effects and sex-specific effects dominated (Table 1). Interestingly, the main effects of mtDNA on life span and survival were manifested only in males: males carrying L mtDNA lived for longer than did those carrying E mtDNA (marginal mean life span 17.17 vs. 15.92 days), while this was not true in females (18.84 vs. 18.88 days). In contrast, nuclear genetic effects were much stronger in females compared to males: females carrying L nDNA lived for longer than did those carrying E nDNA (25.70 vs. 12.01 days), while this was only true to lesser extent in males ( 20.78 vs. 12.32 days). We also found mitonuclear epistatic effects on life span, but these were also sex-specific (Table 1): while males showed little evidence for mitonuclear epistasis for life span, epistasis was more pronounced in females (Fig. 2).

\section{VARIATION IN ETC COMPLEX ACTIVITY}

Mitochondrial bioenergetics was affected by past selection for age at reproduction and this was manifested through both nuclear and mitochondrial genetic effects (Table 2). Moreover, the effects of the nuclear genome upon ETC activity was to a large extent contingent upon the mitochondrial genome, as demonstrated by the significant epistatic interaction terms for all ETC complexes $($ Mito $\times$ Nucl and Mito $\times$ Nucl $\times$ Sex terms in Table 2$)$. We note that the only main mitochondrial effect seen was on the activity of ETC complex II, which is remarkable considering that this complex is built exclusively by nDNA-encoded proteins. Further, a striking facet of variation in ETC activity was the extent to which these effects were sex-specific. Not only were ETC activities generally distinct in mitochondria harvested in males and females (main effect of "Sex" in Table 2), but nuclear, mitochondrial, and epistatic genetic effects were in several cases expressed in distinct ways in males and females (see interaction terms involving Sex in Table 2). These analyses unveiled marked and complex sex specificity of the genetics of mitochondrial bioenergetics. Below, we will comment upon specific aspects of our results.

In general, the pattern of mitonuclear epistasis was to a large extent consistent with expectations based on mitonuclear coadaptation, which predicts that reconstituted MN lines (EE and LL) would share a relatively similar ETC activity that would be higher 
Females
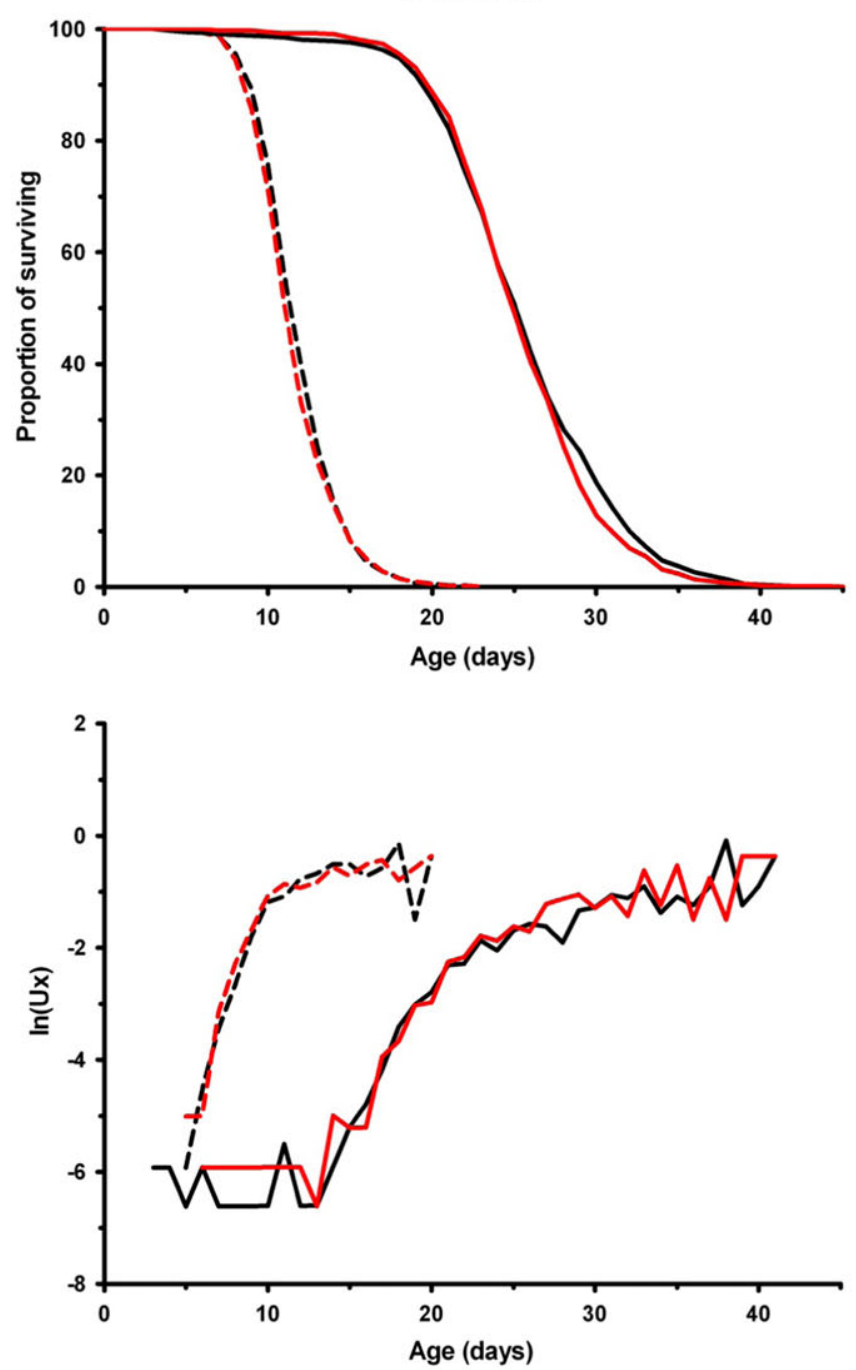
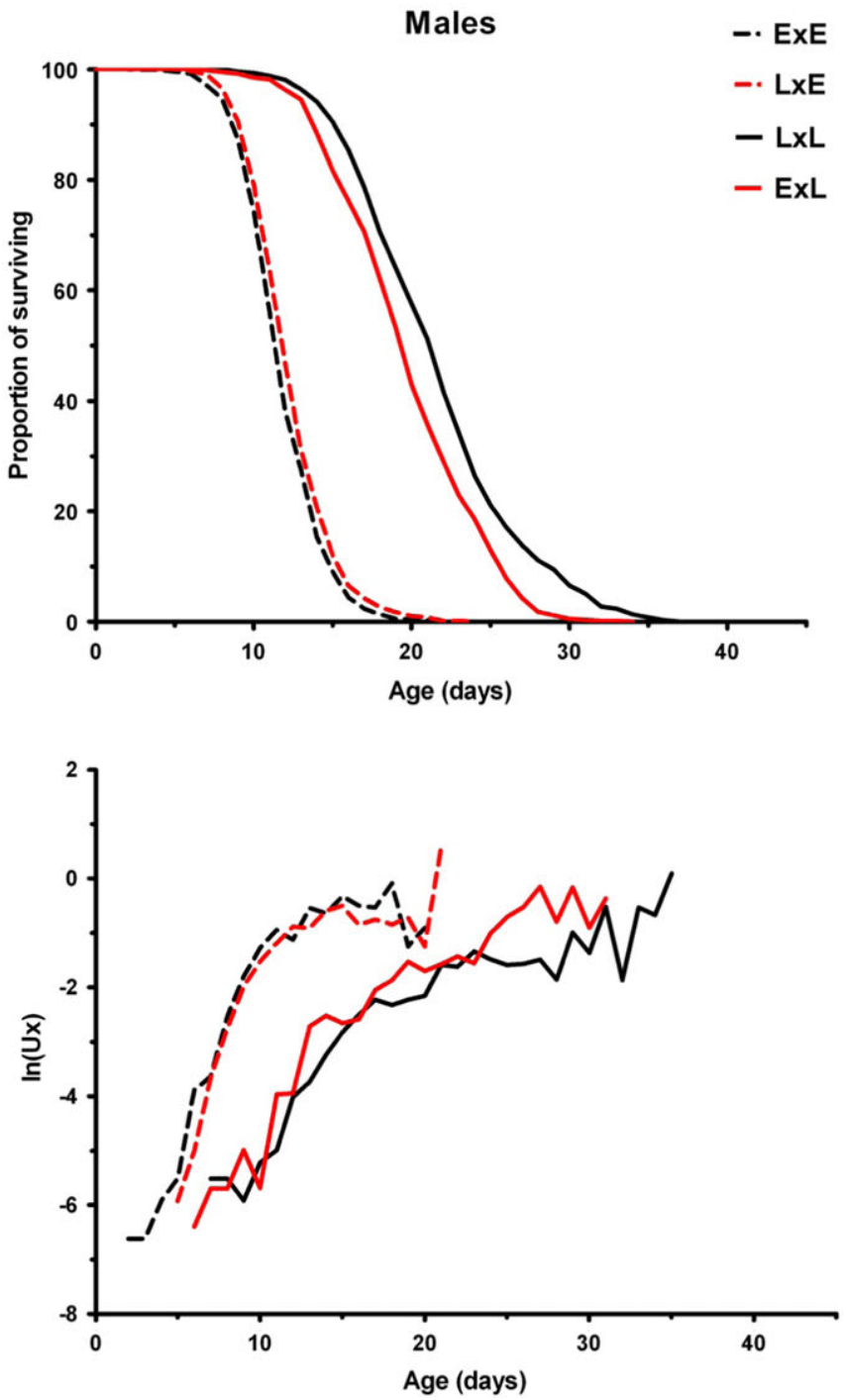

Figure 1. Survivorship and mortality curves (averaged across replicate lines) of virgin females and males from the disrupted (LE and EL; red) and reconstituted (EE and LL; black) mitonuclear lines (denoted as mtDNA x nDNA).

than that exhibited by disrupted MN lines (LE and EL) which, in turn, would be mutually relatively similar (Rand et al. 2004). This pattern was exhibited, for example, by females for ETC complex I and IV (Fig. 3). For ETC complex I, III, and IV, sex-specific ETC activity was lower when a nuclear genome was coexpressed with a mitochondrial genome with which it did not share selection regime in all of the 12 cases and significantly so in 10 cases (Fig. 3). Further, for complex I, the significant sex by mitonuclear interaction (Table 2) supports a stronger coadaptation in females compared to males (Fig. 3). The effects of mitonuclear disruption on ETC complex II were somewhat idiosyncratic and different than that in the other complexes, in that the direction of the effect of mitonuclear disruption was reversed in the $\mathrm{L}$ nuclear genetic background in both sexes (Fig. 3, Table 2).

To better characterize the significant sex-specific effects, we fitted analogous linear mixed models separately for the two sexes.
This verified that the mitonuclear interaction was much stronger in females than in males for ETC complex I ( $F=4.67$ vs. $F=$ $2.72)$, but the reversed was true to a lesser extent for complex II ( $F=0.20$ vs. $F=1.08$ ). For ETC complex IV, the mitochondrial genetic effect was significantly weaker in females than in males ( $F=0.00$ vs. $F=5.69$ ). We note that, for both complex I and IV, the L nuclear background was uniquely unaffected by mtDNA haplotype in males only (Fig. 3), suggesting a component of female-limited mitochondrial epistasis for these particular ETC complexes.

\section{PREDICTING VARIATION IN LIFE-HISTORY TRAITS WITH ETC COMPLEX ACTIVITIES}

We modeled sex-specific variation in five different key life-history traits across the $20 \mathrm{MN}$ lines, using mean activities of the four 
Table 1. The results of $(A)$ a mixed effects Cox proportional hazards model, evaluating the effects of the evolutionary history of the mitochondrial and the nuclear genome, as well as sex upon survival, $(B)$ the results of an analogous model of size specific survival and (C) a generalized linear mixed model of variance in lifespan.

\begin{tabular}{|c|c|c|c|c|c|c|c|}
\hline \multirow[b]{2}{*}{ Source } & \multicolumn{2}{|l|}{$A$} & \multicolumn{2}{|l|}{$B$} & \multicolumn{3}{|l|}{$C$} \\
\hline & $Z$ & $P$ & $Z$ & $P$ & $F$ & $n \mathrm{df}, d \mathrm{df}$ & $P$ \\
\hline Mito & 0.17 & 0.860 & 0.48 & 0.630 & 1.96 & 1,16 & 0.181 \\
\hline Nuclear & 21.99 & $<0.001$ & 14.25 & $<0.001$ & 481.82 & 1,16 & $<0.001$ \\
\hline Sex & 0.02 & 0.980 & 1.52 & 0.130 & 287.44 & 1,5968 & $<0.001$ \\
\hline Mito $\times$ nuclear & 0.48 & 0.630 & 0.99 & 0.320 & 1.37 & 1,16 & 0.258 \\
\hline Mito $\times$ sex & 3.49 & $<0.001$ & 3.64 & $<0.001$ & 41.48 & 1,5968 & $<0.001$ \\
\hline Nuclear $\times \operatorname{sex}$ & 15.44 & $<0.001$ & 12.9 & $<0.001$ & 794.36 & 1,5968 & $<0.001$ \\
\hline Mito $\times$ nuclear $\times$ sex & 1.42 & 0.160 & 1.33 & 0.180 & 8.66 & 1,5968 & 0.003 \\
\hline Weight & & & 17.55 & $<0.001$ & & & \\
\hline Weight $\times$ nuclear & & & 7.6 & $<0.001$ & & & \\
\hline \multirow[t]{2}{*}{ Weight $\times$ sex } & & & 2.45 & 0.014 & & & \\
\hline & Variance & SD & Variance & SD & Variance & SE & \\
\hline Line & 0.054 & 0.232 & 0.082 & 0.286 & 1.168 & 0.430 & \\
\hline
\end{tabular}

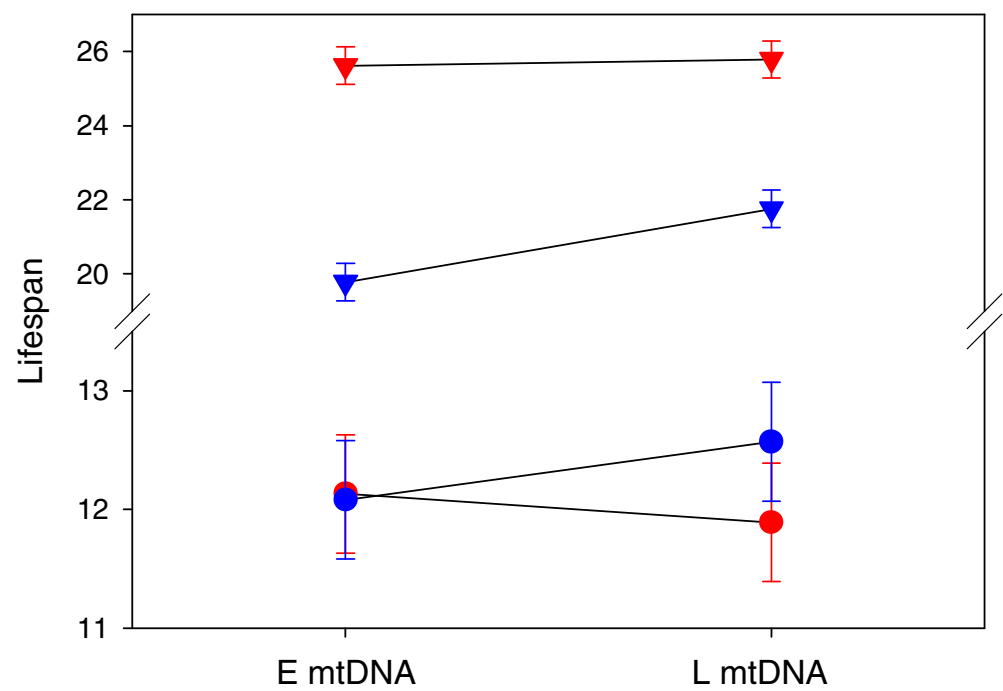

Figure 2. The effects of the evolutionary history of the nuclear and the mitochondrial genome on mean life span ( \pm SE) in virgin females (red) and males (blue). Circles denote nuclear backgrounds from the "early" selection regime (E nDNA) and triangles nuclear backgrounds from the "late" selection regime (L nDNA). Note that the ordinate is broken.

ETC complexes per line and sex as explanatory factors. Overall, these models were highly significant $(P<0.001$ in all cases) and explained a sizeable proportion of variation in life-history traits (Table 3). The addition of the four interaction terms between sex and ETC activity did not collectively significantly improve model fit to data (multiple partial $F$ tests; $P>0.082$ in all five cases), although the sex $\times$ ETCIII activity had a significant effect in isolation on longevity $\left(F_{1,28}=5.65, P=0.024\right)$. Models of the sexes separately showed that ETC activity explained $60-80 \%$ of the variation in mean life-history traits across lines. A common multivariate analysis, naïve to sex, showed a strong canonical correlation between the four ETC activities and the five life-history traits (first canonical axis: $\mathrm{R}=0.89$, Bartlett's test: $\left.\chi_{20}^{2}=75.7, P<0.001\right)$, but also showed that males and females differed somewhat in the multivariate scaling of mitochondrial bioenergetics and life history (Fig. 4). Inspection of canonical loadings on the first canonical axis in analyses of the sexes separately (females: $R=0.92$, Bartlett's test: $\chi_{20}^{2}=40.7$, $P<0.004$; males: $R=0.91$, Bartlett's test: $\chi_{20}^{2}=35.7, P=$ 0.017 ) suggested that this was primarily due to a higher impact of ETC II activity, but a lower of ETC I activity, on life-history variation in females compared to males (Fig. 5). We conclude that variation in life-history traits across the MN lines were linked to variation in mitochondrial bioenergetics and that the pattern of 
Table 2. The results of linear mixed models of the effects of the evolutionary history of mitochondrial and nuclear DNA and sex on the activity of the mitochondrial ETC complexes.

\begin{tabular}{|c|c|c|c|c|c|c|c|c|c|c|c|c|}
\hline \multirow[b]{2}{*}{ Source } & \multicolumn{3}{|c|}{ ETC complex I } & \multicolumn{3}{|c|}{ ETC complex II } & \multicolumn{3}{|c|}{ ETC complex III } & \multicolumn{3}{|c|}{ ETC complex IV } \\
\hline & $\mathrm{df}$ & $F$ & $P$ & df & $F$ & $P$ & df & $F$ & $P$ & df & $F$ & $P$ \\
\hline Mitochondrial & 1,16 & 0.26 & 0.619 & 1,16 & 15.51 & 0.001 & 1,16 & 0.05 & 0.833 & 1,16 & 1.10 & 0.309 \\
\hline Nuclear & 1,16 & 6.42 & 0.022 & 1,16 & 11.59 & 0.004 & 1,16 & 55.43 & $<0.001$ & 1,16 & 0.01 & 0.953 \\
\hline Sex & 1,176 & 5.81 & 0.017 & 1,166 & 66.69 & $<0.001$ & 1,176 & 1.31 & 0.253 & 1,176 & 9.03 & 0.003 \\
\hline Mito $\times$ nuclear & 1,16 & 6.32 & 0.023 & 1,16 & 0.00 & 0.995 & 1,16 & 26.23 & $<0.001$ & 1,16 & 5.68 & 0.030 \\
\hline Mito $\times \operatorname{sex}$ & 1,176 & 0.55 & 0.461 & 1,166 & 0.01 & 0.928 & 1,176 & 3.33 & 0.070 & 1,176 & 21.01 & $<0.001$ \\
\hline Nuclear $\times$ sex & 1,176 & 0.05 & 0.832 & 1,166 & 5.63 & 0.019 & 1,176 & 0.11 & 0.737 & 1,176 & 9.87 & 0.002 \\
\hline \multirow{2}{*}{$\begin{array}{l}\text { Mito } \times \text { nuclear } \times \\
\text { sex }\end{array}$} & 1,176 & 8.22 & 0.005 & 1,166 & 4.97 & 0.027 & 1,176 & 0.11 & 0.739 & 1,176 & 0.00 & 0.999 \\
\hline & & Variance & SE & & Variance & SE & & Variance & SE & & Variance & SE \\
\hline Line & & $1.9 \times 10^{-}$ & $0.8 \times 10^{-3}$ & & $4.03 \times 10^{-5}$ & $1.6 \times 10^{-5}$ & & $1.9 \times 10^{-3}$ & $0.7 \times 10^{-3}$ & & 0.17 & 0.06 \\
\hline
\end{tabular}

this multivariate covariation was somewhat different in males and females.

A striking facet of these modeling efforts was the differences seen in the direction and magnitude of effects between activity and life history across ETC complexes (see Table 3). Although high activities of complexes II and III were significantly associated with longer juvenile development time, higher adult body weight, longer adult life span, a lower rate of senescence, and a slower deceleration of old-age mortality, complex IV and I showed less or even the opposite association with the five life-history traits.

Many of these effects were apparently driven by the effects of mitonuclear disruption on the covariance between ETC complex function and life-history traits. Analogous models including only the EE and LL lines showed significant effects only of variation in ETC complex III activity upon life histories (Table 3). This implies that the larger variance in ETC complex activity that resulted from mitonuclear disruption (Fig. 3) strengthened the covariance between ETC complex activity and life-history traits.

\section{Discussion}

A number of studies have uncovered diverse links between mitochondrial metabolism and the biology of aging and life histories (Balaban et al. 2005; Guarente 2008; Bratic and Larsson 2013). Our study provides several novel insights into the mechanisms through which age-specific selection leads to the evolution of adult longevity and other life-history traits. First, we show that selection for, and the evolution of, a long life is associated with altered bioenergetic properties of mitochondria in these beetles. Variation in life history and ageing were in fact closely associated with ETC activity, especially that of complex III. Second, we provide experimental support for a role of mitonuclear coadaptation in the evolution of mitochondrial bioenergetics. ETC activity was significantly depressed when nuclear genomes were coexpressed with mitochondrial genomes from the opposing selection regime. Third, we were able to disentangle the relative importance that the nuclear and the mitochondrial genomes, as well as their interaction, have in causing the evolution seen. The nDNA effect on ETC activity was large, but there were also direct mtDNA effects and sizeable mitonuclear epistatic effects. Fourth, we show that many of the effects seen were sex-specific, in a manner largely congruent with the fact that males represent a genetic "dead-end" for mtDNA. Next, we will discuss each of these points in some detail.

\section{MITOCHONDRIAL BIOENERGETICS AND AGEING}

Our results provide novel form of support for a link between the evolution of life span and mitochondrial functioning. Although this link has been established by both correlational studies (Melvin and Ballard 2006; Wolff et al. 2016) and a large body of studies using RNAi, genetic screens, or induced mutations (see Balaban et al. 2005), we are unaware of experimental evolution studies that directly demonstrate that selection on life span is associated with evolutionary modulation of OXPHOS functioning. Beetles from $\mathrm{L}$ lines live more than twice as long as those from $\mathrm{E}$ lines (Đorđević et al. 2015). Our results show that the activities of ETC complex I, II, and especially III were higher in beetles from L lines. Multiple regression analyses showed that a high activity of complex II and III and a relatively low activity of complex IV were associated with a long life span and a low rate of ageing across our MN lines. Restricting these analyses to only coevolved mitonuclear combinations (Table 3) suggested that the activity of complex III plays key role. At a first glance, these results may seem counterintuitive: given that complex III is considered to be one of the major sites of production of ROS (Balaban et al. 2005), one might expect that a higher ETC activity should increase ROS production and decrease life span (Harman 1956, 1972). In support, studies of Drosophila (Copeland et al. 2009) and worms (Dillin et al. 2002) have demonstrated a longevity extending effect of complex III subunit knockdown. However, this expectation is dubious for at least three reasons. First, the OXPHOS pathway is a complex cascade and many factors interact, in ways 

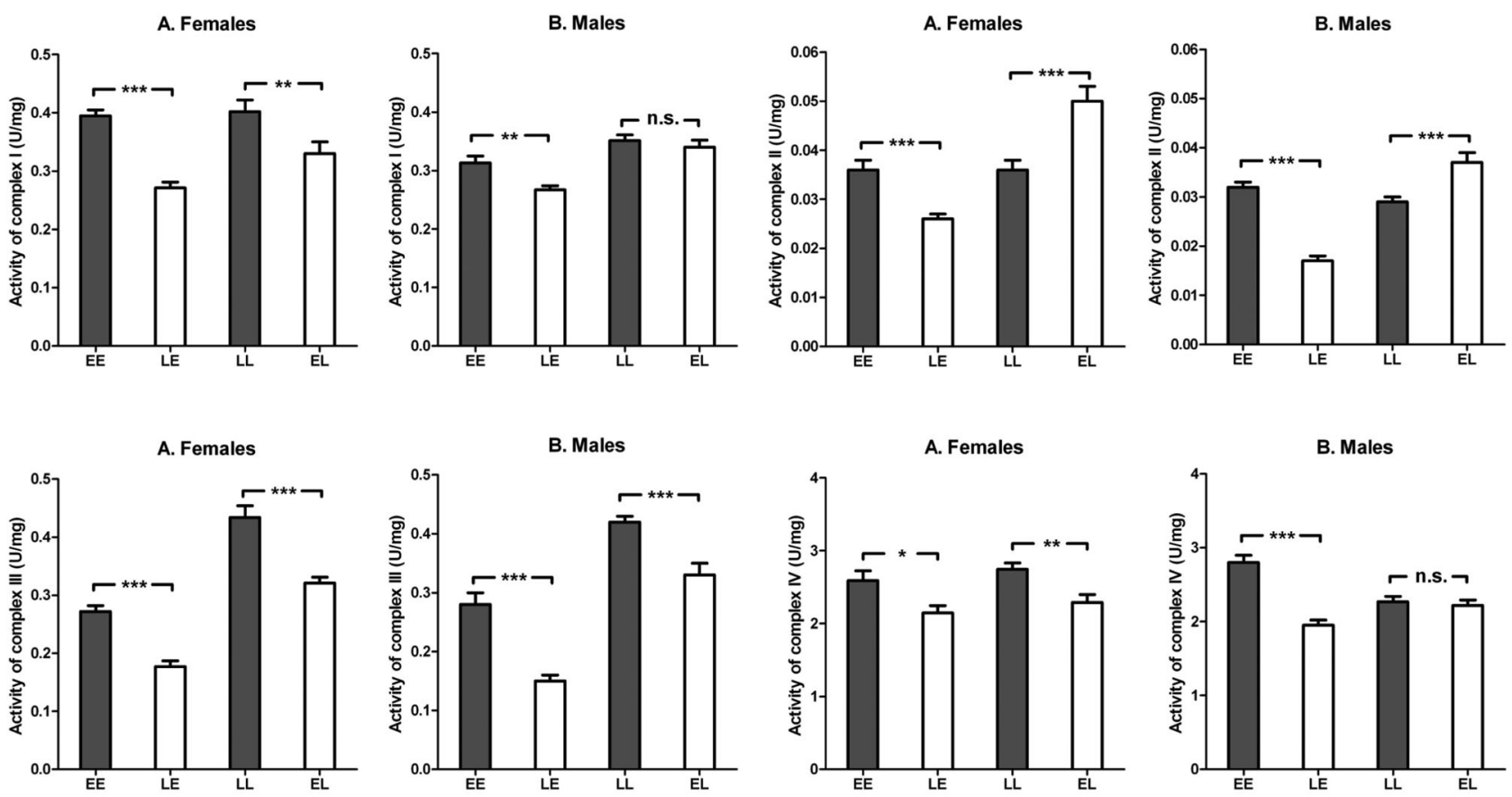

Figure 3. Mean ( \pm SE) activity of the mitochondrial ETC complexes. Shown are sex-specific means for complex I (top left two panels), complex II (top right two panels), complex III (bottom left two panels), and complex IV (bottom right two panels) in adults of Acanthoscelides obtectus with either reconstituted (EE, LL) or disrupted mitonuclear genotypes (LE, EL). Here, the first letter denotes the evolutionary history of mitochondria and the second denotes the evolutionary history of the nuclei ( $E$, selection for early reproduction; $\mathrm{L}$, selection for late reproduction). Shown are also the results of focused $t$ tests of the effects of mitonuclear coadaptation on ETC activity, as manifested by differences between nuclear types expressed with their native (gray bars) versus nonnative (white bars) mitochondria $\left({ }^{*} P<0.05 ;{ }^{* *} P<0.01 ;{ }^{* * *} P<0.001\right)$. See Table 2 for a full statistical evaluation.

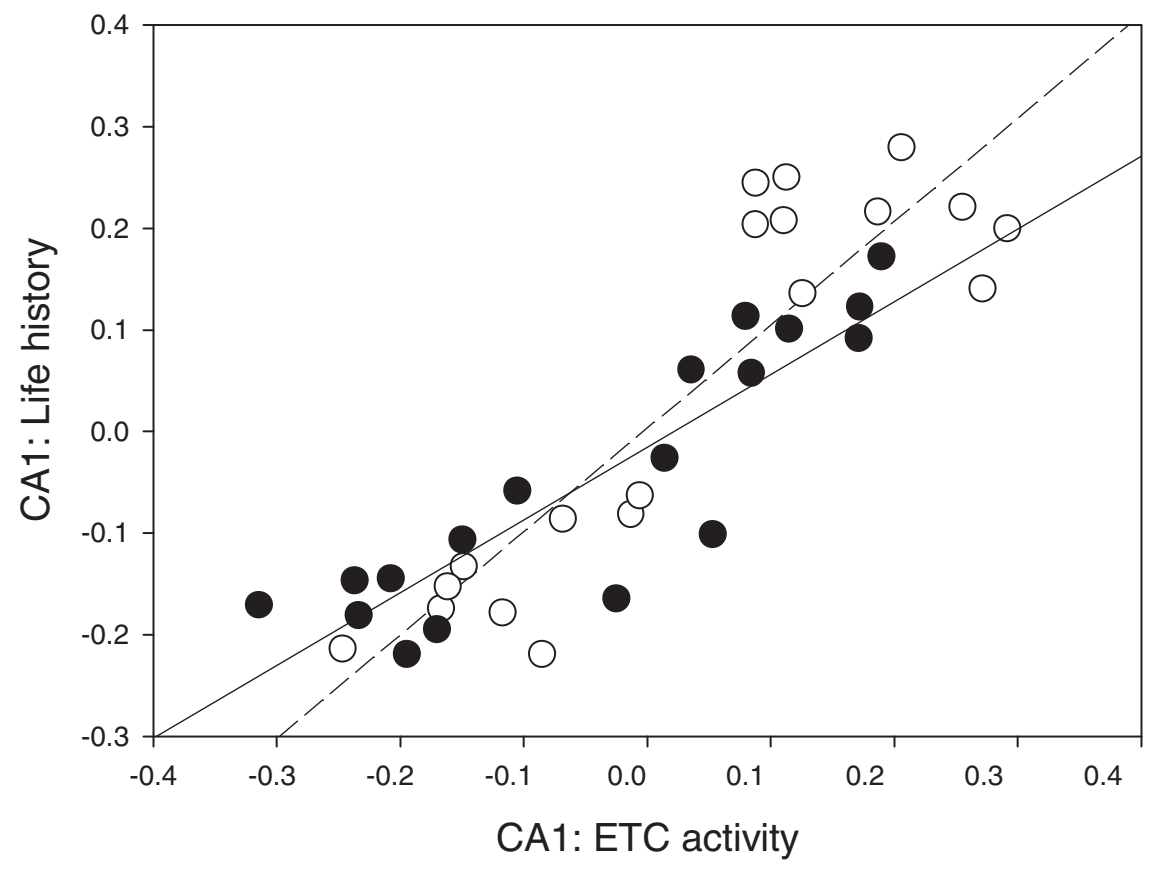

Figure 4. Sex-specific ordination of MN lines along the first canonical axis of covariation between the activity of ETC complexes and life-history traits. Variation in ETC activity explained a large amount of variation in life histories (Stewart-Love Canonical Redundancy Index: 0.55), but the precise scaling differed somewhat between the sexes (resampling test based on $10^{5}$ bootstraps; $P=0.046$ ). Females are here denoted by open circles and a dashed fitted regression line for illustration only, males by closed circles and a solid line. 


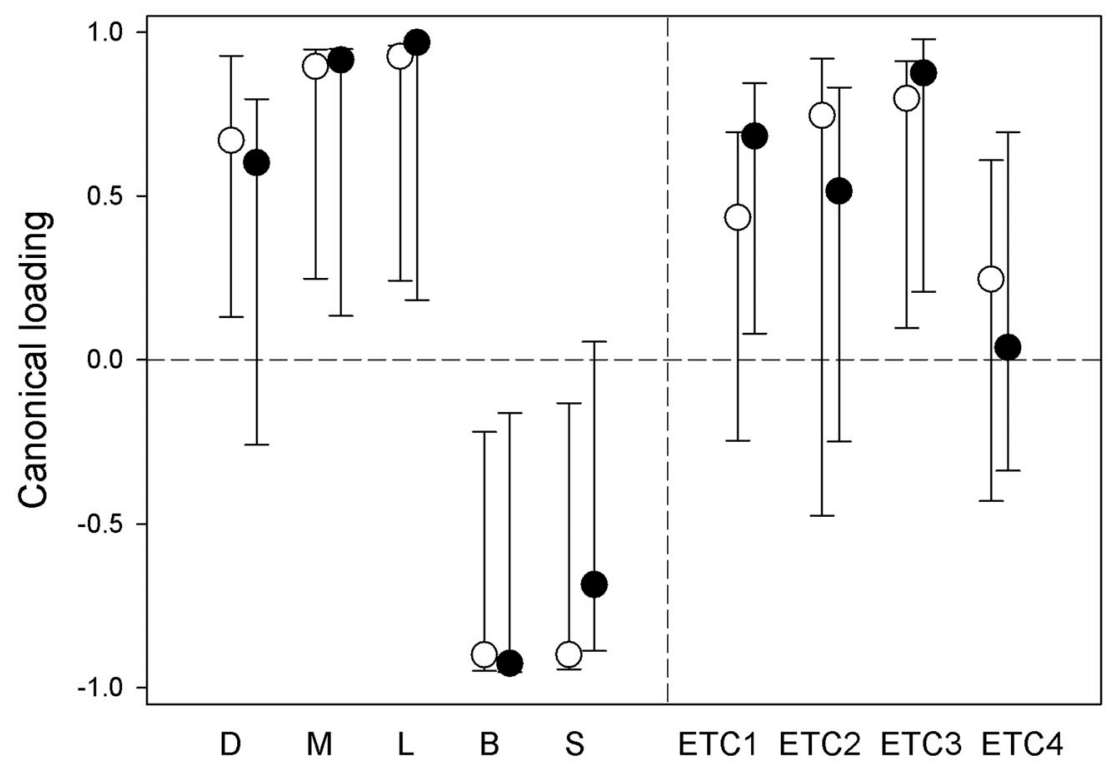

Figure 5. Canonical loadings for both the set of life-history traits (development time, mass, longevity, senescence [B], and old-age mortality [S]; left part) and the set of ETC activities (right part) on the first canonical axis, from canonical correlation analyses performed separately for females (open circles) and males (closed circles). Much of this multivariate covariation was shared between the sexes, although the relative contribution of some of the variables to the latent variables differed somewhat. Shown are also bootstrapped $95 \%$ Cls, based on $10^{3}$ bootstraps corrected for axis reversals.

that are not fully understood, to generate ROS (Balaban et al. 2005). Given this, it is unlikely that relationships between ETC activity of single complexes and ROS production are simple. Second, it has been predicted that a relatively high activity of complex III should be associated with low ROS production and this has some correlational support (Melvin and Ballard 2006). Third, recent studies have shown that ROS are important in many cellular signaling pathways, suggesting that a view of ROS as simply a toxic by-product of mitochondrial respiration is oversimplified (Holzerová and Prokisch 2015). For example, a low level of oxidants is essential for normal metabolome function (Orr et al. 2013). Although we did not measure mitochondrial ROS production, it is possible that higher levels of ROS in L compared to E beetles might even extend life span by stimulating stress pathways (Lane 2011). Beetles from L lines are, indeed, more resistant to oxidative stress (Šešlija et al. 1999; Lazarević et al. 2013). Phenomena such as reduced ROS leakage from mitochondria, elevated antioxidative defense, and increased damage reparation systems have been described in long-lived lines of Drosophila (Kurapati et al. 2000; Arking et al. 2002; Hansen et al. 2012; Orr et al. 2013) and may also be involved in A. obtectus.

It is certainly possible that a higher rate of ubiquinol oxidation at complex III, relative to its reduction at complex I, prevents reverse electron transport and therefore lowers, rather than increases, ROS production at complex I in agreement with the "spare oxidative capacity hypothesis" (Lane 2005; Melvin and Ballard 2006). Increased complex III activity could also be beneficial if metabolic reprogramming of $\mathrm{L}$ beetles toward accumulation of lipids and carbohydrates (Lazarević et al. 2012) involves higher entrance of electrons to the respiratory chain through FAD-linked dehydrogenases that bypass complex I (Lenaz et al. 2007).

Our findings are consistent with the tenet that the OXPHOS pathway stands at the root of ageing-related processes (Balaban et al. 2005; Guarente 2008). Our analysis showed that ETC complex activity not only predicted some $70 \%$ of the variance in longevity and rate of senescence across our MN lines, but was also associated with development rate and body size. We suggest that the fact that we employed long-term selection on life span added both inferential confidence and statistical power, noting that significant within-species correlations between ETC activity and longevity have been documented in some (Melvin and Ballard 2006), but not all (Jumbo-Lucioni et al. 2012) studies of standing genetic variation in Drosophila. In a more general sense, our results underpin the suggestion that mitonuclear genetic variation underlies variation in conglomerated phenotypes such as life-history syndromes (Ballard and Melvin 2010) and behavioral syndromes (Løvlie et al. 2014), and that this is mediated through mitonuclear effects on mitochondrial bioenergetics.

\section{MITONUCLEAR COADAPTATION}

The mitochondrial and nuclear genomes jointly produce the building blocks of the energy-producing OXPHOS and the mitochondrial translational machineries and mitonuclear coadaptation must 
Table 3. The results of analyses of covariance of sex-specific variation across the $20 \mathrm{MN}$ lines, using activity of the ETC complexes to predict life-history traits and demographic parameters.

\begin{tabular}{|c|c|c|c|c|c|c|c|c|c|c|}
\hline \multirow[b]{2}{*}{ Source } & \multicolumn{2}{|c|}{ Development time } & \multicolumn{2}{|c|}{ Body weight } & \multicolumn{2}{|c|}{ Longevity } & \multicolumn{2}{|c|}{ Senescence $(b)$} & \multicolumn{2}{|c|}{ Old-age mortality $(s)$} \\
\hline & $F_{1,32}$ & {$\left[F_{1,12}\right]$} & $F_{1,32}$ & {$\left[F_{1,12}\right]$} & $F_{1,32}$ & {$\left[F_{1,12}\right]$} & $F_{1,31}$ & {$\left[F_{1,12}\right]$} & $F_{1,31}$ & {$\left[F_{1,12}\right]$} \\
\hline ETC I & 0.06 & {$[0.35]$} & 0.00 & {$[0.59]$} & 0.07 & {$[0.19]$} & 0.15 & {$[0.01]$} & 0.22 & [0.13] \\
\hline ETC II & $16.90^{+}$ & {$[0.05]$} & $7.82^{+}$ & {$[0.41]$} & $12.40^{+}$ & {$[0.22]$} & $10.44^{-}$ & {$[0.32]$} & $10.52^{-}$ & {$[0.59]$} \\
\hline ETC III & $4.27^{+}$ & {$\left[9.50^{+}\right]$} & $28.01^{+}$ & {$[20.76+]$} & $\mathbf{3 5 . 9 3}^{+}$ & {$\left[15.99^{+}\right]$} & $33.84^{-}$ & {$[20.13-]$} & $11.66^{-}$ & {$\left[7^{3} .35^{-}\right]$} \\
\hline ETC IV & $16.58^{-}$ & {$[3.23]$} & $6.60^{-}$ & {$[1.71]$} & $11.86^{-}$ & {$[1.57]$} & $11.63^{+}$ & {$[0.99]$} & 2.40 & {$[0.12]$} \\
\hline Sex & 0.30 & [2.38] & 6.34 & {$[3.50]$} & 1.32 & {$[0.84]$} & 4.98 & {$[0.36]$} & 6.28 & {$[0.33]$} \\
\hline$R^{2}$ & 0.56 & {$[0.65]$} & 0.70 & [0.76] & 0.73 & [0.69] & 0.71 & [0.71] & 0.58 & {$[0.44]$} \\
\hline
\end{tabular}

Effects are given as $F$ values and significant effects $(P<0.05)$ are given in bold. Within square brackets are the results of analogous models including only the 10 reconstituted MN lines (i.e., EE and LL). ${ }^{+}$Significant positive effect; ${ }^{-}$Significant negative effect.

thus be central in the evolution of mitochondrial bioenergetics (Rand et al. 2004; Willett and Burton 2004; Ballard and Rand 2005; Levin et al. 2014). Direct within-species evidence for this comes from the analysis of mtDNA backcross lines. For example, in the marine copepod Tigriopus californicus, the coexpression of between-population combinations of mitonuclear types are associated with decreased ETC complex activity (Rawson and Burton 2002; Ellison and Burton 2006). This accords with the general prediction that an experimental mismatch of coadapted partners should result in depressed ETC activity (Rand et al. 2004), as a result of defects in complex assembly and/or impaired regulation of transcription and translation of mitochondrial proteins (Burton et al. 2013). Two aspects of our results support the hypothesis that life-history evolution in A. obtectus has involved mitonuclear coadaptation of the OXPHOS pathway. First, the general depression of ETC complex activity observed for disrupted mitonuclear genotypes (Fig. 3) are in line with general predictions as well as previous empirical observations (Rawson and Burton 2002; Rand et al. 2004; Ellison and Burton 2006; Burton et al. 2013). Second, the sex-specific mitonuclear epistatic effects on life span and activity of ETC complex I are consistent with the prediction that mitonuclear coadaptation should be more pronounced in females compared to males (see below). Collectively, thus, our results are consistent with mitonuclear coadaptation, adding to a growing body of evidence that mitonuclear allelic interactions affect the evolution of the life-history phenotypes (reviewed by Dobler et al. 2014; Levin et al. 2014).

\section{NUCLEAR, MITOCHONDRIAL, AND MITONUCLEAR GENETIC EFFECTS}

Our experimental design allowed us to elucidate the relative roles of nDNA, mtDNA, and their interaction in the divergent evolution seen in ETC complex activity. Considering the fact that mtDNA encodes for 13 proteins, while the remaining proteins $(>1000)$ in the mitochondrial proteome are encoded by the nuclear genome, it is not surprising that the nuclear genome had the largest overall effects on ETC complex activity. Future next-generation sequencing efforts will be aimed at identifying the nuclear loci responsible for this evolutionary response. What is perhaps more surprising is the fact that the effects of mtDNA haplotype were almost as large, especially for the activity of ETC complex II and IV, although mtDNA effects have been demonstrated in $D$. melanogaster (Maklakov et al. 2006; Correa et al. 2012). The effects seen on complex II are likely indirect, as this is composed exclusively of nDNA encoded proteins. Recent studies show that the activity of complex II, which is part of both the (the Krebs cycle) TCA cycle and ETC, is particularly sensitive to several metabolic sensors and regulators which, in turn, are broadly affected by the bioenergetic properties of the mitochondrion (Pfleger et al. 2015). For example, in Caenorhabditis elegans, downregulation of complex I leads to an upregulation of complex II (Pujol et al. 2013; Dancy et al. 2014). In Drosophila, Villa-Cuesta et al. (2014) showed mtDNA effects on the activity of complex II and metabolomic profiling suggested that these effects were mediated through the actions of other mitochondrially encoded proteins. It is thus possible that the increased complex II activity seen in EL beetles represents compensatory response to mitochondrial malfunction. The mtDNA effects seen on ETC complex IV activity, which were largely sex-specific (see below), are interesting in that they are congruent with (1) the fact that the cytochrome c oxidase subunit I (COI) haplotype is indeed different across E and L lines (Stojković et al. 2016) and (2) studies of mtDNA variation showing that $\mathrm{COI}$ is enriched with nonsynonymous substitutions in A. obtectus across its range (Oliveira et al. 2013).

Mitochondrial genetic effects are often expressed as epistatic interactions with the nuclear genome (see Dobler et al. 2014) and this was true in our case as well. Significant mitonuclear epistatic effects, whether sex-specific or not, were partly responsible for the evolutionary divergence seen in ETC activity for all 
four complexes. We note that the epistatic effects were proportionally largest for ETC complex I, which is well aligned with the fact that the mtDNA contributes with no less than seven of some 37 proteins that collectively form complex I. The fact that $A . o b$ tectus is a nonmodel species currently precludes the identification of the interacting genes, something which future sequencing efforts might enable.

A previous analysis of mtDNA haplotype frequencies in our selection lines implicated mtDNA as an important mediator of divergent evolution of life span and life history in A. obtectus (Stojković et al. 2016). By providing direct evidence for mtDNA effects on the OXPHOS pathway, the current study represents a mechanistic validation of this inference.

\section{SEX-SPECIFIC EFFECTS}

We found that ETC activity was generally higher in females than in males, a common finding in many other species as well, which may reflect the metabolic requirements of egg production (Ballard et al. 2007b; Wolff et al. 2016). However, the fact that mtDNA is maternally inherited creates an interesting sexual asymmetry for response to selection on mitochondrial genes and their interactions with nuclear genes (Frank and Hurst 1996; Gemmell et al. 2004). On the one hand, because mtDNA haplotypes with sex-limited deleterious effects in males are hidden from purifying selection and can persist, we generally expect larger mtDNA effects in males than in females (Innocenti et al. 2011; Dobler et al. 2014). On the other hand, mitonuclear coevolution can be expected to be more evident in females compared to males. Mitonuclear genotypes only cosegregate through females and selection for male-specific compensatory nuclear mutations should be relatively ineffectual, because such mutations are only under selection in males, are only beneficial in a specific mitochondrial genetic background, and because selection on male-specific compensatory mutations is bounded by the fitness effects of mitochondrial haplotypes (Wade 2014; Immonen et al. 2016). This predicts that the rate of compensatory evolution in males is only about one-fourth of the rate of spread of female-benefit mitonuclear genotypes (Wade 2014). Our data provide support for both of these predictions. First, the main effect of mtDNA on survival and life span were significantly stronger in males than females and the same was true for activity of ETC complex IV. Second, the mitonuclear epistatic effects on life span and activity of ETC complex I was significantly stronger in females. Moreover, the pattern of the effect of mitonuclear disruption on ETC activity was more closely aligned with expectations based on mitonuclear coadaptation in females compared to males, especially for complex I.

In summary, the sex-specific genetic effects observed in $A$. obtectus were largely in accord with predictions based on sexspecific optima of life span and life history (i.e., sexual conflict; Stojković et al. 2011; Maklakov and Lummaa 2013) and an "edge" for females due to maternal inheritance of mtDNA (Wade 2014). Interestingly, Stojković and Savković (2011) highlighted the fact that selection on life-history traits should be sexually antagonistic in $\mathrm{L}$ lines but less so in $\mathrm{E}$ lines, and a more pronounced sexual dimorphism in $\mathrm{L}$ lines compared to $\mathrm{E}$ lines have been reported for longevity (Stojković and Savković 2011; Stojković et al. 2011), oxidative stress resistance (Lazarević et al. 2013), and metabolite content (Lazarević et al. 2012). We found this to be true for ETC activity as well, as sexual dimorphism in LL lines were higher than in EE lines for all complexes (Storer's SD index; ETC I: -0.14 vs. -0.12 ; ETC II: -0.22 vs. -0.12 ; ETC III: -0.04 vs. 0.02; ETC IV: -0.19 vs. 0.08).

We also found that the precise multivariate relationship between ETC activity and life-history traits differed somewhat between the sexes. Such differences are predicted for sex-specific fitness components, such as gonad function (Immonen et al. 2015), and the fact that this was true for shared life-history traits highlights the point that the genetic architecture for physiological and life-history traits is often sex-specific (Poissant et al. 2010).

In conclusion, the OXPHOS pathway lies at the heart of life-history evolution, simply because fitness is ultimately built by metabolic conversion of resources acquired from the environment into offspring. By using lines selected for a long or a short life, we were able to show that the evolution of life span and life-history traits is closely associated with modulation of the activity of all ETC subunits in A. obtectus. Moreover, the evolution of ETC activity was to a large extent due to epistatic interactions between the nuclear and the mitochondrial genome and showed marked sex specificity, where mitonuclear coadaptation was more evident in females. Our results not only underpin the link between mitochondrial functioning and life-history evolution, but also provide support for the promotion of mtDNA to an important agent of adaptive evolution.

\section{ACKNOWLEDGMENTS}

This contribution was supported by the European Research Council (GENCON AdG-294333), the Swedish Research Council (621-20144523), and the Serbian Ministry of Education, Science and Technological Development (grant no. 173007). The authors declare that they have no conflict of interest.

\section{Accession Numbers}

All relevant data are within the article and its Supporting Information files. All sequence data are available from GenBank, accession numbers KU168414-KU168416.

\section{LITERATURE CITED}

Arking, R., S. Buck, D. S. Hwangbo, and M. Lane. 2002. Metabolic alternations and shifts in energy allocations are corequisites for the expression of extended longevity genes in Drosophila. Ann. NY Acad. Sci. 959:251-262. 
Arnqvist, G., D. K. Dowling, P. Eady, L. Gay, T. Tregenza, M. Tuda, and D. J. Hosken. 2010. Genetic architecture of metabolic rate: environment specific epistasis between mitochondrial and nuclear genes in an insect. Evolution 64:3354-3363.

Balaban, R. S., S. Nemoto, and T. Finkel. 2005. Mitochondria, oxidants, and aging. Cell 120: 483-495.

Ballard, J. W. O., and R. G. Melvin. 2010. Linking the mitochondrial genotype to the organismal phenotype. Mol. Ecol. 19:1523-1539.

Ballard, J. W. O., and N. Pichaud. 2014. Mitochondrial DNA: more than an evolutionary bystander. Funct. Ecol. 28:218-231.

Ballard, J. W. O., and D. M. Rand. 2005. The population biology of mitochondrial DNA and its phylogenetic implications. Annu. Rev. Ecol. Evol. Syst. 36:621-642.

Ballard, J. W. O., R. G. Melvin, S. D. Katewa, and K. Maas. 2007a. Mitochondrial DNA variation is associated with measurable differences in life-history traits and mitochondrial metabolism in Drosophila simulans. Evolution 61:1733-1747.

Ballard, J. W. O., R. G. Melvin, J. T. Miller, and S. D. Katewa. 2007b. Sex differences in survival and mitochondrial bioenergetics during aging in Drosophila. Aging Cell 6:699-708.

Barrinetos, A. 2002. In vivo and in organello assessment of OXPHOS activities. Methods 26:307-316.

Bradford, M. M. 1976. A rapid and sensitive method for the quantitation of microgram quantities of protein utilizing the principle of protein-dye binding. Anal. Biochem. 72:248-254.

Bratic, A., and N. G. Larsson. 2013. The role of mitochondria in aging. J. Clin. Invest. 123:951-957.

Burton, R. S., R. J. Pereira, and F. S. Barreto. 2013. Cytonuclear genomic interactions and hybrid breakdown. Ann. Rev. Ecol. Evol. Syst. 44:281302.

Clancy, D. J. 2008. Variation in mitochondrial genotype has substantial lifespan effects which may be modulated by nuclear background. Aging Cell 7:795-804.

Copeland, J. M., J. Cho, T. Lo Jr., J. H. Hur, S. Bahadorani, T. Arabyan, J. Rabie, J. Soh, W. David, and D. W. Walker. 2009. Extension of Drosophila life span by RNAi of the mitochondrial respiratory chain. Curr. Biol. 19:1591-1598.

Correa, C. C., W. C. Aw, R. G. Melvin, N. Pichaud, and J. W. O. Ballard. 2012. Mitochondrial DNA variants influence mitochondrial bioenergetics in Drosophila melanogaster. Mitochondrion 12:459464.

Dancy, B. M., M. M. Sedensky, and P. G. Morgan. 2014. Effects of the mitochondrial respiratory chain on longevity in C. elegans. Exp. Gerontol. $56: 245-255$.

Das, J. 2006. The role of mitochondrial respiration in physiological and evolutionary adaptation. Bioassays 28:890-901.

Dillin, A., A. L. Hsu, N. Arantes-Oliveira, J. Lehrer-Graiwer, H. Hsin, A. G. Fraser, R. S. Kamath, J. Ahringer, and C. Kenyon. 2002. Rates of behavior and aging specified by mitochondrial function during development. Science 298:2398-2401.

Dobler, R., B. Rogell, F. Budar, and D. K. Dowling. 2014. A meta-analysis of the strength and nature of cytoplasmic genetic effects. J. Evol. Biol. 27:2021-2034.

Đorđević, M., U. Savković, J. Lazarević, N. Tucić, and B. Stojković. 2015. Intergenomic interactions in hybrids between short-lived and long-lived lines of a seed beetle: analyses of life history traits. Evol. Biol. 42:461472.

Dowling, D. K., A. A. Maklakov, U. Friberg, and F. Hailer. 2009. Applying the genetic theories of ageing to the cytoplasm: cytoplasmic genetic covariation for fitness and lifespan. J. Evol. Biol. 22:818827.
Ellison, C. K., and R. S. Burton. 2006. Disruption of mitochondrial function in interpopulation hybrids of Tigriopus californicus. Evolution 60:13821391.

Frank, S. A., and L. D. Hurst. 1996. Mitochondria and male disease. Nature 383:224.

Gallach, M., and E. Betran. 2011. Intralocus sexual conflict resolved through gene duplication. Trends Ecol. Evol. 26:222-228.

Gallach, M., C. Chandrasekaran, and E. Betran. 2010. Analyses of nuclearly encoded mitochondrial genes suggest gene duplication as a mechanism for resolving intralocus sexually antagonistic conflict in Drosophila. Genome Biol. Evol. 2:835-850.

Gemmell, N. J., Z. J. Metcalf, and F. W. Allendorf. 2004. Mother's course: the effect of mtDNA on individual fitness and population viability. Trends Ecol. Evol. 19:238-244.

Guarente, L. 2008. Mitochondria — a nexus for aging, calorie restriction, and sirtuins? Cell 132:171-176.

Hansen, T. Ø., P. Sarup, V. Loeschcke, and S. I. S. Rattan. 2012. Agerelated and sex-specific differences in proteasome activity in individual Drosophila flies from wild type, longevity-selected and stress resistant strains. Biogerontology 13:429-438.

Harman, D. 1956. Aging: a theory based on free radical and radiation chemistry. J. Gerontol. 11:298-300.

- 1972. The biologic clock: the mitochondria. J Am. Geriatr. Soc. 20:145-147.

Holzerová, E., and H. Prokisch. 2015. Mitochondria: much ado about nothing? How dangerous is reactive oxygen species production? Int. J. Biochem. Cell B 63:16-20.

Houtkooper, R. H., L. Mouchiroud, D. Ryu, N. Moullan, E. Katsyuba, G. Knott, R. W. Williams, and J. Auwerx. 2013. Mitonuclear protein imbalance as a conserved longevity mechanism. Nature 497:451-457.

Immonen, E., J. Rönn, C. Watson, D. Berger, and G. Arnqvist. 2015. Complex mitonuclear interactions and metabolic costs of mating in male seed beetles. J. Evol. Biol. 29:360-370.

Immonen, E., M. Collet, J. Goenaga, and G. Arnqvist. 2016. Direct and indirect genetic effects of sex-specific mitonuclear epistasis on reproductive ageing. Heredity 116:338-347.

Innocenti, P., E. H. Morow, and D. K. Dowling. 2011. Experimental evidence supports a sex-specific selective sieve in mitochondrial genome evolution. Science 332:845-848.

James, A. C., and J. W. O. Ballard. 2003. Mitochondrial genotype affects fitness in Drosophila simulans. Genetics 164:187-194.

Janssen, A. J. M., F. J. M. Trijbels, R. C. A. Sengers, J. A. M. Smeitink, L. P. van den Heuvel, L. T. M. Wintjes, B. J. M. Stoltenborg-Hogenkamp, and R. J. T. Rodenburg. 2007. Spectrophotometric assay for complex I of the respiratory chain in tissue samples and cultured fibroblasts. Clin. Chem. 53:729-734.

Jumbo-Lucioni, P., S. Bu, S. T. Harbison, J. C. Slaughter, T. F. C. Mackay, D. R. Moellering, and M. De Luca. 2012. Nuclear genomic control of naturally occurring variation in mitochondrial function in Drosophila melanogaster. BMC Genomics 13:659.

Kageyama, D., S. Narita, T. Imamura, and A. Miyanoshita. 2010. Detection and identification of Wolbachia endosymbionts from laboratory stocks of stored-product insect pests and their parasitoids. J. Stored Prod. Res. 46:13-19.

Kurapati, R., H. B. Passananti, M. R. Rose, and J. Tower. 2000. Increased hsp22 RNA levels in Drosophila lines genetically selected for increased longevity. J. Gerontol. 55A:B552-B559.

Lane, N. 2005. Power, sex, suicide: mitochondria and the meaning of life. Oxford Univ. Press, New York.

. 2011. Mitonuclear match: optimizing fitness and fertility over generations drives ageing within generations. BioEssays 33:860-869. 
Lazarević, J., N. Tucić, D. Šešlija-Jovanović, J. Večeřa, and D. Kodrík. 2012. The effects of selection for early and late reproduction on metabolite pools of Acanthoscelides obtectus Say. Insect Sci. 19:303-314.

Lazarević, J., M. Đorđević, B. Stojković, and N. Tucić. 2013. Resistance to prooxidant agent paraquat in the short- and long-lived lines of the seed beetle (Acanthoscelides obtectus). Biogerontology 14:141152.

Lemos, B., L. O. Araripe, and D. L. Hartl. 2008. Polymorphic Y chromosomes harbor cryptic variation with manifold functional consequences. Science 319:91-93.

Lenaz, G., R. Fato, G. Formiggini, and M. L. Genova. 2007. The role of Coenzyme Q in mitochondrial electron transport. Mitochondrion 7S:S8S33.

Levin, L., and D. Mishmar. 2015. A genetic view of the mitochondrial role in ageing: killing us softly. Adv. Exp. Med. Biol. 847:89-106.

Levin, L., A. Blumberg, G. Barshad, and D. Mishmar. 2014. Mito-nuclear coevolution: the positive and negative sides of functional ancient mutations. Front. Genet. 5:448.

Li, Y. Y., P. G. Fields, B. P. Pang, P. C. Coghlin, and K. D. Floate. 2015. Prevalence and diversity of Wolbachia bacteria infecting insect pests of stored products. J. Stored Prod. Res. 62:93-100.

Luo, C., J. Long, and J. Liu. 2008. An improved spectrophotometric method for a more specific and accurate assay of mitochondrial complex III activity. Clin. Chim. Acta 395:38-41.

Løvlie, H., E. Immonen, E. Gustavsson, E. Kazancioğlu, and G. Arnqvist. 2014. The influence of mitonuclear genetic variation on personality in seed beetles. Proc. R. Soc. Lond. B. 281:20141039.

Maklakov, A. A., and V. Lummaa. 2013. Evolution of sex differences in lifespan and aging: causes and constraints. BioEssays 35:717-724.

Maklakov, A. A., U. Friberg, D. K. Dowling, and G. Arnqvist. 2006. Withinpopulation variation in cytoplasmic genes affect female life span and aging in Drosophila melanogaster. Evolution 60:2081-2086.

Meiklejohn, C. D., K. L. Montooth, and D. M. Rand. 2007. Positive and negative selection on the mitochondrial genome. Trends Genet. 23:259263.

Melvin, R. G., and J. W. O. Ballard. 2006. Intraspecific variation in survival and mitochondrial oxidative phosphorylation in wild-caught Drosophila simulans. Aging Cell 5:225-233.

O’Neill, S. L., R. Giordano, M. Colbert, T. L. Karr, and H. M. Robertson. 1992. 16S rRNA phylogenetic analysis of the bacterial endosymbionts associated with cytoplasmic incompatibility in insects. Proc. Natl. Acad. Sci. USA 89:2699-2702.

Oliveira, M. R. C., A. S. Corrêa, G. A. de Souza, R. N. C. Guedes, and L. O. de Oliveira. 2013. Mesoamerican origin and pre-and post-Columbian expansions of the ranges of Acanthoscelides obtectus Say, a cosmopolitan insect pest of the common bean. PloS One 8:e70039.

Orr, W. C., S. N. Radyuk, and R. S. Sohal. 2013. Involvement of redox state in the aging of Drosophila melanogaster. Antioxid. Redox. Signal 19:788-803.

Pfleger, J., M. He, and M. Abdellatif. 2015. Mitochondrial complex II is a source of the reserve respiratory capacity that is regulated by metabolic sensors and promotes cell survival. Cell Death Dis. 6:e1835.

Pletcher, S. D. 1999. Model fitting and hypothesis testing for age-specific mortality data. J. Evol. Biol. 12:430-439.

Poissant, J., A. J. Wilson, and D. W. Coltman. 2010. Sex-specific genetic variance and the evolution of sexual dimorphism: a systematic review of cross-sex genetic correlations. Evolution 64:97-107.

Pujol, C., I. Bratić-Hench, M. Šumaković, J. Hench, A. Mourier, L. Baumann, V. Pavlenko, and A. Trifunović. 2013. Succinate dehydrogenase upregulation destabilize complex I and limits the lifespan of gas-7 mutant. PloS One 8:e59493.
Rand, D. M., A. G. Clark, and L. M. Kann. 2001. Sexually antagonistic cytonuclear fitness interactions in Drosophila melanogaster. Genetics 159:173-187.

Rand, D. M., R. A. Haney, and A. J. Fry. 2004. Cytonuclear coevolution: the genomics of cooperation. Trends Ecol. Evol. 19:645-653.

Rand, D. M., A. Fry, and L. Sheldahl. 2006. Nuclear-mitochondrial epistasis and Drosophila aging: introgression of Drosophila simulans mtDNA modifies longevity in D. melanogaster nuclear background. Genetics 172:329-341.

Rawson, P. D., and R. S. Burton. 2002. Functional coadaptation between cytochrome $\mathrm{c}$ and cytochrome $\mathrm{c}$ oxidase within allopatric populations of a marine copepod. Proc. Natl. Acad. Sci. USA 99:12955-12958.

Schlötterer, C., R. Kofler, E. Versace, R. Tobler, and S. U. Franssen. 2015. Combining experimental evolution with next-generation sequencing: a powerful tool to study adaptation from standing genetic variation. Heredity 114:431-440.

Šešlija, D., D. Blagojević, M. Spasić, and N. Tucić. 1999. Activity of superoxide dismutase and catalase in the bean weevil (Acanthoscelides obtectus) selected for postponed senescence. Exp. Gerontol. 34:185-195.

Šešlija Jovanović, D., M. Đorđević, U. Savković, and J. Lazarević. 2014. The effect of mitochondrial complex I inhibitor on longevity of short-lived and long-lived seed beetles and its mitonuclear hybrids. Biogerontology 15:487-501.

Stojković, B., and U. Savković. 2011. Gender differences in longevity in early and late reproduced lines of the seed beetle. Arch. Biol. Sci. (Belgrade) 63:129-136.

Stojković, B., D. Šešlija Jovanović, J. Perovanović, and N. Tucić. 2011. Sexual activity and reproductive isolation between age-specific selected populations of seed beetle. Ethology 117:812-821.

Stojković, B., A. Sayadi, M. Đorđević, J. Jović, U. Savković, and G. Arnqvist. 2016. Divergent evolution of lifespan associated with mitochondrial DNA evolution. Evolution (In press).

Therneau, T. 2015. coxme: Mixed Effects Cox Models. R package version 2.25. Available at http://CRAN.R-project.org/package=coxme. Accessed February 27, 2016.

Tucić, N., I. Gliksman, D. Šešlija, D. Milanović, S. Mikuljanac, and O. Stojković. 1996. Laboratory evolution of longevity in the bean weevil (Acanthoscelides obtectus). J. Evol. Biol. 9:485-503.

Tucić, N., O. Stojković, I. Gliksman, D. Milanović, and D. Šešlija. 1997. Laboratory evolution of life-history traits in the bean weevil (Acanthoscelides obtectus): the effects of density-dependent and age-specific selection. Evolution 51:1896-1909.

Villa-Cuesta, E., M. A. Holmbeck, and D. M. Rand. 2014. Rapamycin increases mitochondrial efficiency by mtDNA-dependent reprogramming of mitochondrial metabolism in Drosophila. J. Cell Sci. 127:2282-2290.

Wade, M. J. 2014. Paradox of mother's curse and the maternally provisioned offspring microbiome. Cold Spring Harb. Perspect. Biol. 6:a017541.

Willett, C. S., and R. S. Burton. 2004. Evolution of interacting proteins in the mitochondrial electron transport system in a marine copepod. Mol. Biol. Evol. 21:443-453.

Wolff, J. N., E. D. Ladoukakis, J. A. Enríquez, and D. K. Dowling. 2014. Mitonuclear interactions: evolutionary consequences over multiple biological scales. Philos. Trans. R. Soc. B 369:20130443.

Wolff, J. N., N. Pichaud, M. F. Camus, G. Côté, P. U. Blier, and D. K. Dowling. 2016. Evolutionary implications of mitochondrial genetic variation: mitochondrial genetic effects on OXPHOS respiration and mitochondrial quantity change with age and sex in fruit flies. J. Evol. Biol. J. Evol. Biol. 29:736-747.

Associate Editor: T. Flatt Handling Editor: R. Shaw 


\section{Supporting Information}

Additional Supporting Information may be found in the online version of this article at the publisher's website:

Table S1. Mean development time, body mass, and longevity for female and male seed beetles in different mitonuclear lines and grand means for reconstituted (EE, LL) and disrupted mitonuclear genotypes (LE, EL).

Table S2. Parameters of the logistic survival function, $\ln (\mathrm{a}), \mathrm{b}$, and s, for female and male seed beetles in different mitonuclear lines and means $( \pm \mathrm{SE})$ for reconstituted (EE, LL) and disrupted mitonuclear genotypes (LE, EL).

Table S3. Mean $( \pm \mathrm{SE})$ activity of the mitochondrial ETC complexes I-IV in virgin females and males from the disrupted (LE and EL) and reconstituted (EE and LL) mitonuclear lines.

Figure S1. Survival and mortality curves for virgin females and males of all MN lines with disassociated (LE and EL; red) and reconstituted (EE and LL; black) mitonuclear genotypes. 